\title{
Feature saliency and feedback information interactively impact visual category learning
}

\author{
Rubi Hammer ${ }^{1,2,3}$ *, Vladimir Sloutsky ${ }^{4}$ and Kalanit Grill-Spector ${ }^{1,5}$ \\ ${ }^{1}$ Department of Psychology, Stanford University, Stanford, CA, USA \\ 2 Department of Communication Sciences and Disorders, Northwestern University, Evanston, IL, USA \\ ${ }^{3}$ Interdepartmental Neuroscience Program, Northwestern University, Evanston, IL, USA \\ ${ }^{4}$ Department of Psychology and Center for Cognitive Science, The Ohio State University, Columbus, OH, USA \\ ${ }^{5}$ Stanford Neuroscience Institute, Stanford University, Stanford, CA, USA
}

\section{Edited by:}

Petko Kusev, Kingston University London, UK

\section{Reviewed by:}

Jonathan R. Folstein, Florida State University, USA

Renata Melinda Heilman,

Babes-Bolyai University, Romania Paulo Carvalho, Indiana University, USA

\section{*Correspondence:}

Rubi Hammer, Developmental Cognitive Neuroscience Lab, Department of Communication Sciences and Disorders,

Northwestern University, 2240

Campus Drive, Evanston,

IL 60208-2952, USA

e-mail: rubi.hammer@north

western.edu
Visual category learning (VCL) involves detecting which features are most relevant for categorization. VCL relies on attentional learning, which enables effectively redirecting attention to object's features most relevant for categorization, while 'filtering out' irrelevant features. When features relevant for categorization are not salient, VCL relies also on perceptual learning, which enables becoming more sensitive to subtle yet important differences between objects. Little is known about how attentional learning and perceptual learning interact when $\mathrm{VCL}$ relies on both processes at the same time. Here we tested this interaction. Participants performed VCL tasks in which they learned to categorize novel stimuli by detecting the feature dimension relevant for categorization. Tasks varied both in feature saliency (low-saliency tasks that required perceptual learning vs. high-saliency tasks), and in feedback information (tasks with mid-information, moderately ambiguous feedback that increased attentional load, vs. tasks with high-information non-ambiguous feedback). We found that mid-information and high-information feedback were similarly effective for VCL in high-saliency tasks. This suggests that an increased attentional load, associated with the processing of moderately ambiguous feedback, has little effect on VCL when features are salient. In low-saliency tasks, VCL relied on slower perceptual learning; but when the feedback was highly informative participants were able to ultimately attain the same performance as during the high-saliency VCL tasks. However, VCL was significantly compromised in the low-saliency mid-information feedback task. We suggest that such lowsaliency mid-information learning scenarios are characterized by a 'cognitive loop paradox' where two interdependent learning processes have to take place simultaneously.

Keywords: category learning, categorization, attentional learning, perceptual learning, visual perception, feedback processing, feature saliency, perceptual expertise

\section{INTRODUCTION}

The human brain is capable of managing effectively an immense amount of visual information, rendering it rapidly into a reliable and meaningful representation of objects and events. The cognitive process enabling this is Visual Category Learning (VCL), which involves the detection of object features that are most relevant for categorization. In many learning scenarios VCL requires supervision that may involve the processing of labeled exemplars (Lupyan etal., 2007; Davis etal., 2012; but see also Sloutsky etal., 2007), or being informed that few objects are from the same-category or from different categories without the use of labels (Namy and Gentner, 2002; Hammer et al., 2009a,b; Mathy etal., 2013). Deducing associations between category labels and exemplars, or the categorical relation between few objects, can be accomplished by feedback that follows the learner decision, indicating whether the decision was correct or not (Love, 2002; Maddox et al., 2003; Daniel and Pollmann, 2010; Antzoulatos and Miller, 2011; Lopez-Paniagua and Seger, 2011).

In everyday life scenarios, information provided by feedback during VCL may often be suboptimal, or even misleading. Thus, multiple learning experiences are required to ultimately enable categorizing objects based only on those attributes that are most relevant for categorization (Hammer et al., 2007, 2008). Another challenge characterizing some VCL scenarios is low-saliency and poor representation of features that are relevant for categorization, which may result in such features being overlooked. Facing these challenges, VCL relies on two fundamental processes. The first process is attentional learning, which enables the volitional allocation of attention to relevant features, while 'filtering out' distracting salient features that have little relevance for categorization (Rehder and Hoffman, 2005; Sloutsky and Fisher, 2008; Blair et al., 2009; Hoffman and Rehder, 2010; Sloutsky, 2010; McColeman et al., 2014). The second process is perceptual learning, which enables becoming more sensitive to subtle, initially hard to detect differences between objects from different categories (Shiffrin and Schneider, 1977; Goldstone, 1994, 1998; Goldstone et al., 2001; Roelfsema et al., 2010). Although previous studies showed that both attentional learning and perceptual learning contribute to the reduction of categorization errors, to-date no study systematically tested VCL tasks in which these two processes have to take place simultaneously. 
Attentional learning involves improving the ability to allocate attention to important sensory information, within a given context, while ignoring task-irrelevant information (Nosofsky, 1984; Nosofsky and Palmeri, 1996; Kruschke and Blair, 2000; Sloutsky and Fisher, 2008; Smith et al., 2010). This often results in a rivalry between bottom-up processes where salient features attract more attention than less salient ones (Itti et al., 1998), and top-down processes driven by prior knowledge and expectations regarding which stimuli features have been found to be important in the past (Koch and Tsuchiya, 2007; Baluch and Itti, 2011; but see also Awh et al., 2012). These two attention processes are likely based on two primary brain networks: (i) The ventral attention network acts as a bottom-up saliency detection system enabling the involuntarily reorientation of attention to unexpected salient stimuli in the environment (Corbetta et al., 2008; Vossel et al., 2014). (ii) The dorsal attention network enables short-term circulation of information that is with current subjective importance, and top-down volitional direction of attention to chosen stimulus features (LaBar et al., 1999; Awh and Jonides, 2001). The nature of the interaction between these two attention brain networks is not fully understood. However, this interaction is thought to depend on context, the maturation of prefrontal executive brain regions, and subjective experiences (Gazzaley and Nobre, 2012; Weissman and Prado, 2012; Hammer et al., submitted).

Perceptual learning often results in increased sensitivity and long-lasting improvement in the ability to respond to previously undetected or poorly represented features (Ahissar and Hochstein, 1997; Goldstone, 1998; Fahle and Poggio, 2002). There is an ongoing debate regarding the role of top-down attention control in mediating visual perceptual learning. Presently, most behavioral studies suggest that effective perceptual learning requires intentional direction of attention to the features being learned (Ahissar and Hochstein, 1993; Schoups et al., 2001). Such attentional control may be mediated by corrective feedback that enables the perceiver to realize that two stimuli that were initially confused as being the same are in fact different. In turn, this process may trigger attentional search for visual features that enables differentiating between objects and object categories, eventually resulting in an increased sensitivity to these features (Herzog and Fahle, 2002; Aberg and Herzog, 2012). Others suggest that in some scenarios stimulus-reward pairing may result in "task-irrelevant" perceptual learning, or unintended changes in stimuli representation that may affect later behavior. This may happen when an 'unattended task-irrelevant' sensory feature is being associated with a rewarding outcome (Seitz et al., 2009; Roelfsema et al., 2010).

The perceived feature saliency, and thus the degree to which an object attribute attracts attention, may be altered due to changes in representation such as those that follow perceptual learning. On the other hand, perceptual learning may require effective topdown attention control enabling prolonged focusing of attention to a specific feature (Goldstone and Steyvers, 2001; Serences and Yantis, 2006; Seitz et al., 2009). Arguably, when the objects of interest are complex and differ in multiple low-saliency features, VCL is less likely to be effective without informative guidance (Hammer, submitted). These suggest that perceptual learning and attentional learning do not only affect VCL independently, but they may have a complex context-dependent interaction, where the two learning processes rely on one another and cannot take place at the same time, effectively. Here we tested this interaction by systematically manipulating feature saliency and feedback information. We expected that low-saliency of features relevant for categorization would increase the reliance of VCL on perceptual learning. We expected that ambiguous feedback would hinder VCL by reducing the odds that attention would be exclusively directed to the task-relevant feature dimension in successive learning trials. Consequently, VCL tasks with low-saliency features and ambiguous feedback would rely both on perceptual learning and attentional learning, where the two processes have to take place simultaneously. Such scenarios, where two interdependent learning processes have to take place simultaneously, may involve a 'cognitive loop paradox' with a distinct negative impact on VCL.

We define feature saliency in terms of the physical dissimilarity between objects along a given feature dimension, in a given context (Diesendruck et al., 2003; Hammer and Diesendruck, 2005; Chen et al., 2013). For example, when categorizing Dobermans (large dogs) and Chihuahuas (small dogs), body-size is a highsaliency feature dimension due to salient dissimilarities in body size between these two categories of dogs. On the other hand, when categorizing Labradors and Labradoodles (both are mid-large size dogs), body-size is a low-saliency feature dimension due to high similarities in body size between the two categories of dogs. Note that when two stimuli are perceived as substantially dissimilar from one another across a particular feature dimension, this feature dimension may attract more attention and may a-priori be considered as having higher diagnostic value than a low-saliency feature dimension (Rosch and Mervis, 1975; Tversky, 1977; Nosofsky, 1986; Kruschke, 2003; Chin-Parker and Ross, 2004).

In the current study, in each VCL task stimuli differed from one another in three feature dimensions (e.g. the body width, limbs shape and horns thickness of novel creature-like stimuli) where only one feature dimension was relevant for categorization. In each of the VCL tasks we kept feature saliency similar across the three feature dimensions, making them similarly likely to be a-priori perceived as relevant for categorization. We contrasted between high-saliency VCL tasks and low-saliency VCL tasks, investigating how feedback information is being used in these two learning scenarios. High-saliency tasks simulate scenarios where the categorized objects may differ in several salient attributes, only some of which are important for categorization. Low-saliency tasks simulate scenarios where a greater degree of perceptual expertise is required for detecting fine yet important differences between objects. We argue that in this later scenario, introducing highly informative feedback would be critical for VCL to be effective.

We determined the quantity of feedback information as the degree of ambiguity in each learning trial, where ambiguous feedback did not provide all the information needed for decisive detection of the task-relevant feature dimension in a single learning trial. Ambiguous feedback should not be confused with an inherently incorrect or misleading feedback. Here, in each trial two stimuli were presented simultaneously and the participant had to decide if the two were from the same-category 
or from different-categories. In high-information learning trials, same-category pairs were identical in the task-relevant feature dimension and differed in the two irrelevant feature dimensions, whereas different-categories pairs were different only in the task-relevant feature dimension and were identical in the two irrelevant feature dimensions. This enabled detecting the task-relevant feature dimension using the feedback from a single trial. In midinformation learning trials same-category stimuli were identical in the task-relevant feature dimension but also in one of the irrelevant feature dimensions. In different-categories trials the two stimuli were different in the task-relevant feature dimension and one of the irrelevant feature dimensions. Across multiple trials, the objective category relation between paired stimuli (as it could be deduced from the feedback) was not consistently associated with the irrelevant feature dimensions, but was consistently associated with the relevant feature dimension. Thus, inferring the relevant feature dimension (the categorization rule) was feasible but required integrating information across more trials. These principles are illustrated in Figure 1.

We hypothesized that ambiguous feedback would significantly compromise VCL in low-saliency conditions. In such conditions perceptual learning is more likely to be required not only for increasing sensitivity to important between categories differences, but also for improving the capacity to detect such fine differences, to begin with. In low-saliency VCL conditions features relevant for categorization are not well represented in sensory cortices and thus are likely to be left unattended during early learning trials, specifically when other features may initially be perceived as similarly important (Nosofsky and Palmeri, 1996; Goldstone, 1998; Goldstone etal., 2001). We expected that in such low-saliency conditions ambiguity in feedback would result in prolonged and extensive effort for detecting and isolating task-relevant feature dimensions, since this has to take place simultaneously with becoming more sensitive to differences between stimuli in these feature dimensions (Treisman and Gelade, 1980; Borji and Itti, 2013). In fact, frequently switching attention between several low-saliency feature-dimensions across learning trails, due to ambiguous feedback, can in some extreme scenarios utterly block perceptual learning. Such frequent attention switching may hinder the buildup of brain activation associated with the task-relevant features, preventing it from reaching the threshold required for significant changes in representation to take place (Goldstone, 1994; Gilbert et al., 2001; Kruschke, 2001).

On the other hand, we hypothesized that salient differences between stimuli would be easily detected. In such conditions it is possible to value the importance of each feature dimension by systematic volitional switching of attention between feature dimensions, even when the feedback is ambiguous. It is important to clarify that we expect ambiguous feedback to increase attentional load regardless of features saliency; but, we suggest that when features relevant for categorization are with high-saliency, and the feedback is not too ambiguous, such an increase in attentional load would not be necessarily evident in compromised VCL performances, or would have only small negative effect on VCL. When a feature dimension differentiating between categories would be with low-saliency yet the feedback would be informative, attention could be effectively directed to this feature dimension in multiple successive learning trials, enabling effective perceptual learning. We suggest that lowfeature saliency is always expected to increase the dependency on perceptual learning, and thus would always inhibit VCL. Nevertheless, when the available feedback is informative, VCL would become reasonably manageable even under some low-saliency conditions.

\section{MATERIALS AND METHODS PARTICIPANTS}

Sixty paid adults (36 females), with normal or corrected to normal vision, participated in the experiment. Participants gave informed written consent in accordance with a protocol approved by the Stanford University Institutional Review Board.

\section{EOUIPMENT AND SETTING}

Psychtoolbox $\left(\right.$ MATLAB $^{\circledR}$ ) was used for stimuli presentation on a $1920 \times 1200$ pixels computer display and for the recording of participants' responses. Participants' heads were located about $70 \mathrm{~cm}$

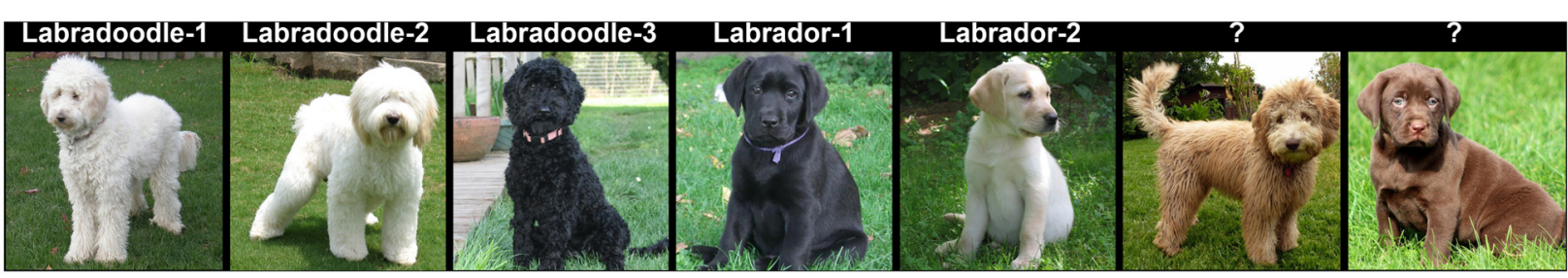

FIGURE 1 | Learning to categorize puppies by comparing and contrasting paired-examples. The most salient feature dimensions differentiating the seven puppies are fur color and fur length. Being informed that two puppies are from the same category can be useful for detecting which feature dimension is most important for categorization. For example, comparing Labradoodle-3 with Labradoodle-1 or Labradoodle-2, or Labrador-1 with Labrador-2, while being informed about their categorical relation, is informative for learning that salient differences in fur color are not important for categorizing these dogs, and thus fur length is more likely to be important. On the other hand, comparing Labradoodle-1 with Labradoodle-2 does not enable inferring which feature is most important. Being informed that two puppies are from distinct categories is also useful for $\mathrm{VCL}$, enabling detecting differences between categories. For example, comparing Labradoodle-3 with Labrador-1, or Labrador-2 with Labradoodle-1 or Labradoodle-2, is informative by highlighting the between categories differences in fur length. On the other hand, comparing Labradoodle-3 with Labrador-2, or Labrador-1 with Labradoodle-1 or Labradoodle-2, is less informative since such comparison is less constraining, enabling deducing that fur color and/or fur length are important for categorization. The labeled examples allow learning that fur length is most relevant for categorization, which in turn allows properly categorizing the two rightmost puppies despite their distinct color (see Hammer et al., 2008 for a formal discussion). 
( $\sim 2$ feet) from the computer screen such that each one of the two simultaneously presented stimuli occupied approximately $14^{\circ}$ of the visual field.

\section{STIMULI}

We used four distinct sets of novel creature-like stimuli. In each set stimuli varied in three feature dimensions, produced from one standard object and three morph-target objects. Each morphtarget differed from the standard in one feature dimension (e.g., shape of head or limb size). Stimuli were generated by morphing between the standard object and each of the three morph-targets using morphing values with steps of $11 \%(0 \%$ to $99 \%$; total of 10 morph levels within each feature dimension). For the experimental VCL tasks, from each stimuli set eight stimuli were used in each saliency-condition so that stimuli differed in three binary feature dimensions. In VCL tasks with high-feature saliency the selected stimuli differed from one another in high morph values within each feature dimension (77\% or higher). In low-saliency VCL tasks stimuli differed in substantially lower morph values (33\% or lower). The selection of stimuli to be used in the experiment was determined based on pilot tests assuring that within each stimuli-set differences in the three feature dimensions were similarly likely to be detected when contrasting between paired stimuli that differed in a single feature dimension. Low-saliency differences were initially less likely to be detected (see Results for pre-learning performances). Due to the nature of stimuli (complex $3 \mathrm{D}$ renderings), fully equalizing feature saliency across the three feature dimensions and across all stimuli sets was not practicable, but the use of four stimuli sets and the counterbalancing across conditions insured that this would not have significant impact on the results (Figure 2; Appendix A in the online supplemental).

In each VCL task there were two predetermined categories. Stimuli in each category had an identical value on one feature dimension (the diagnostic task-relevant feature dimension) and differed in the other two (irrelevant) feature dimensions.

\section{DESIGN}

Experimental conditions differed in feature saliency (high-saliency vs. low-saliency) and two primary levels of feedback information (high-information vs. mid-information). In addition to these four experimental conditions, we tested participants in two types of control tasks: No-information tasks where feedback was provided but it was not informative, and unsupervised categorization tasks with no feedback. These allowed assessment of the contribution of feedback information to VCL in the primary experimental conditions. The control VCL tasks also varied in levels of feature saliency.

Each participant performed four VCL tasks in four pseudorandomly selected conditions (out of four possible experimental conditions and four possible control conditions). For each participant, each VCL task was based on a different stimulus set, and thus the participant had no prior experience with the stimuli at the beginning of each task. Tasks were counterbalanced so that each one of the four stimuli sets was used a similar number of times in each one of the four experimental conditions and four control conditions.

Each VCL task included seven blocks of 24 trials each, where four test-blocks (T1, T2, T3, T4) alternated with three learningblocks (L1, L2, L3). In each trial two creatures were presented simultaneously for 2.2 seconds during which the participant had to decide if the two belong to the same category or to different categories by pressing one of two keys. This was followed by 0.8 seconds inter-trial interval during which the feedback was presented. In learning-blocks in the high-information and midinformation experimental conditions, and the no-information control condition, a green square indicated a correct categorization decision and a red square an incorrect/error decision. In the unsupervised 'learning-blocks,' and in the test-blocks, a yellow square indicated an on-time response (Figure 3).

Participants were instructed that in each VCL task they have to categorize the creatures into two distinct subspecies based on one critical attribute (the task-relevant feature dimension), which can be inferred from the feedback. Participants were told that if they find the feedback to be ineffective, they should guess which feature dimension is most likely to be task-relevant. Participants were told that two creatures of the same subspecies should be identical in this feature dimension, and two creatures from distinct subspecies should differ in this feature dimension. Prior

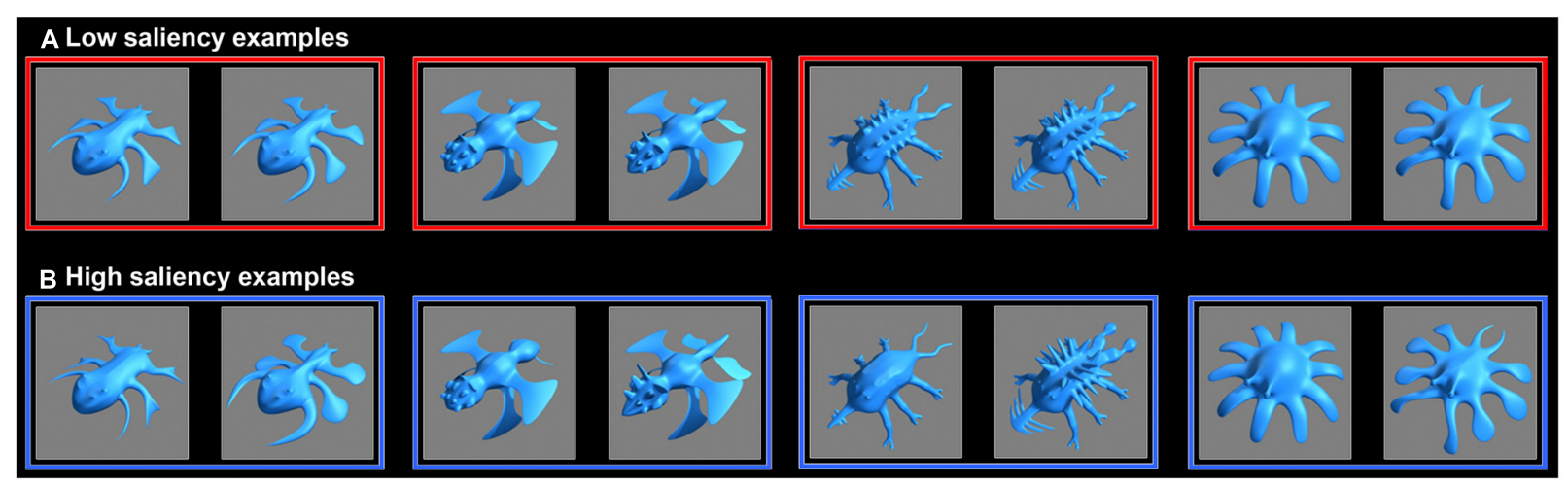

FIGURE 2 | Stimuli examples. Low-saliency pairs (A) and corresponding high-saliency pairs (B) from each one of the four stimuli sets. Each paired stimuli differ in all three varying feature dimensions (see also Appendix $A$ in the online supplemental). 


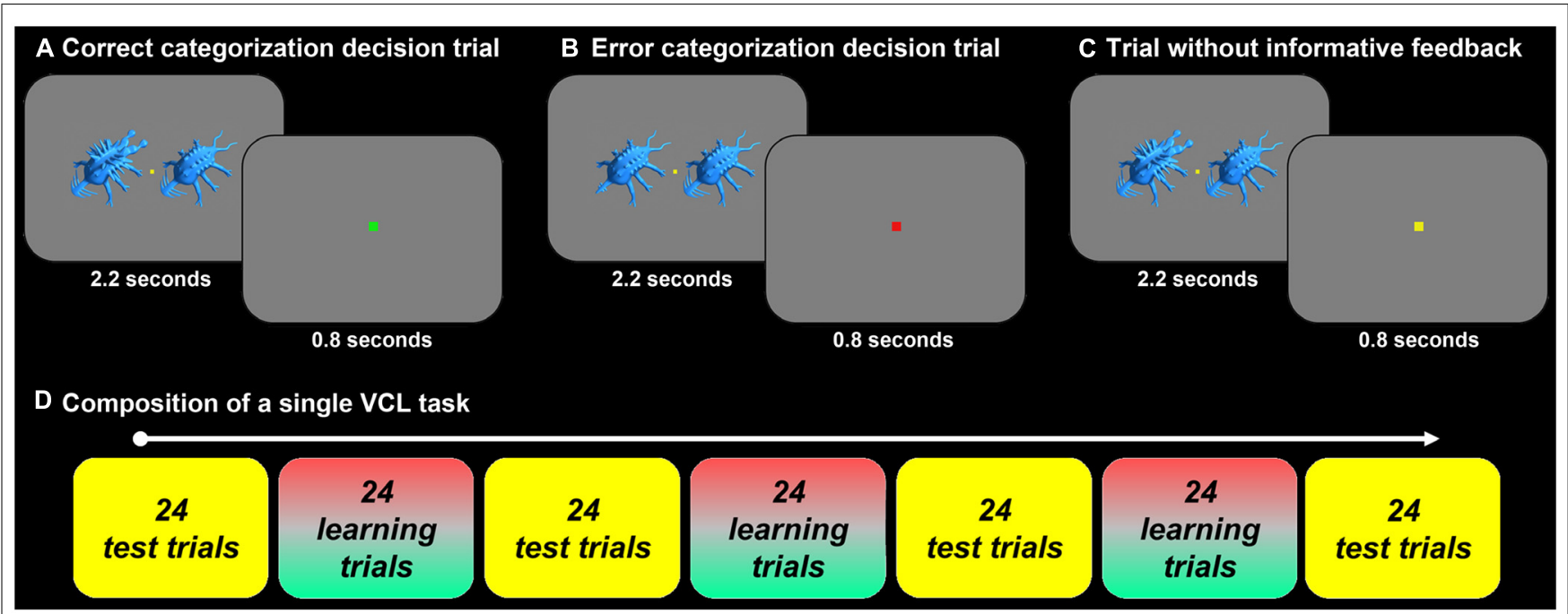

FIGURE 3 | (A) An illustration of a learning trial with feedback indicating correct categorization decision (signified to the participant by a small green square presented after the execution of the categorization decision and stimuli offset). In each trial paired stimuli were presented for 2.2 seconds, during which the participant had to decide if the two creatures were from the same-category or different-categories. Feedback was presented during the following 0.8 seconds. (B) An illustration of a learning trial with feedback indicating incorrect categorization decision (signified by a small red square). (C) An illustration of a trial without informative feedback (a small yellow square signified an on-time response). Such trials were in the test-blocks and in the 'learning-blocks' in the unsupervised categorization control tasks. (D) Schematics of the composition of a single VCL task. Each VCL tasks lasted 8.5 minutes and included four test-blocks that alternated with three learning-blocks. In the unsupervised categorization tasks there were only seven test blocks. Each block included 24 trials. to performing the experimental tasks, the participant performed short warm-up tasks simulating the experimental conditions she was expected to perform (using dedicated stimuli sets that were not used in the experimental tasks). This allowed familiarizing participants with the experimental setting (but not with the stimuli used for the experimental tasks). The duration of each VCL task was 8.5 minutes, with a few minutes break between tasks. The overall duration of an experimental session was 60-75 minutes.

In high-saliency VCL tasks both the within-category and between-categories differences were with high-saliency (Figure 4A), and in low-saliency tasks both the withincategory and between-categories differences were with lowsaliency (Figure 4B).

In learning trials with high-information feedback, paired samecategory creatures were identical in the relevant feature dimension and differed in the two irrelevant feature dimensions (upper pair in Figure 4C). Paired different-categories creatures differed in the relevant feature dimension and were identical in the two irrelevant ones (lower pair in Figure 4C). That is, each highinformation learning trial either indicated all the within-category variability (same-category pairs), or pinpointed the diagnostic feature dimension differentiating between-categories (differentcategories pairs). High-information learning trials can be also formulated as trials in which the task-relevant feature dimension and the irrelevant feature dimensions were anti-correlated (correlation of -1 ): Where ' $A$ ' denotes the relevant feature dimension, ' $B$ ' and ' $C$ ' the irrelevant feature dimensions and ' $X$ ' the categorization decision outcome, the only possible inferred trialby-trial causality in the high-information condition was (four representative trials): $(A \rightarrow X) \cap(A \rightarrow X) \cap(A \rightarrow X) \cap$ $(A \rightarrow X) \cdots$

In the learning trials with mid-information feedback, paired same-category creatures were identical in the relevant feature dimension and one of the two irrelevant feature dimensions (randomly alternating between the two across different trials; upper pair in Figure 4D). Paired different-categories creatures differed in the relevant feature dimension and one of the irrelevant feature dimensions (again, alternating between the two across trials; lower pair in Figure 4D). That is, in each trial there was a degree of ambiguity regarding which feature dimension is relevant for categorization, forcing the use of information from several trials in order to confidently learn the categorization rule. Mid-information learning trials can be also formulated as trials in which there was partial correlation between the task-relevant feature dimension and each one of the two irrelevant feature dimensions: $(A \cup B \rightarrow X) \cap(A \cup C \rightarrow X) \cap(A \cup C \rightarrow X) \cap$ $(A \cup B \rightarrow X) \cdots$

In the learning trials with no-information feedback, paired same-category creatures were identical in all feature dimensions (upper pair in Figure 4E), whereas paired different-categories creatures differed in all feature dimensions (lower pair in Figure 4E). Here the degree of ambiguity was such that the categorization rule could not be inferred even by integrating information across an infinite number of trials. No-information learning trials can be formulated as trials in which the correlation between the task-relevant feature dimension and the two irrelevant feature dimensions is maximized $(+1)$. In such scenarios decisively inferring that a single feature dimension is relevant for categorization 


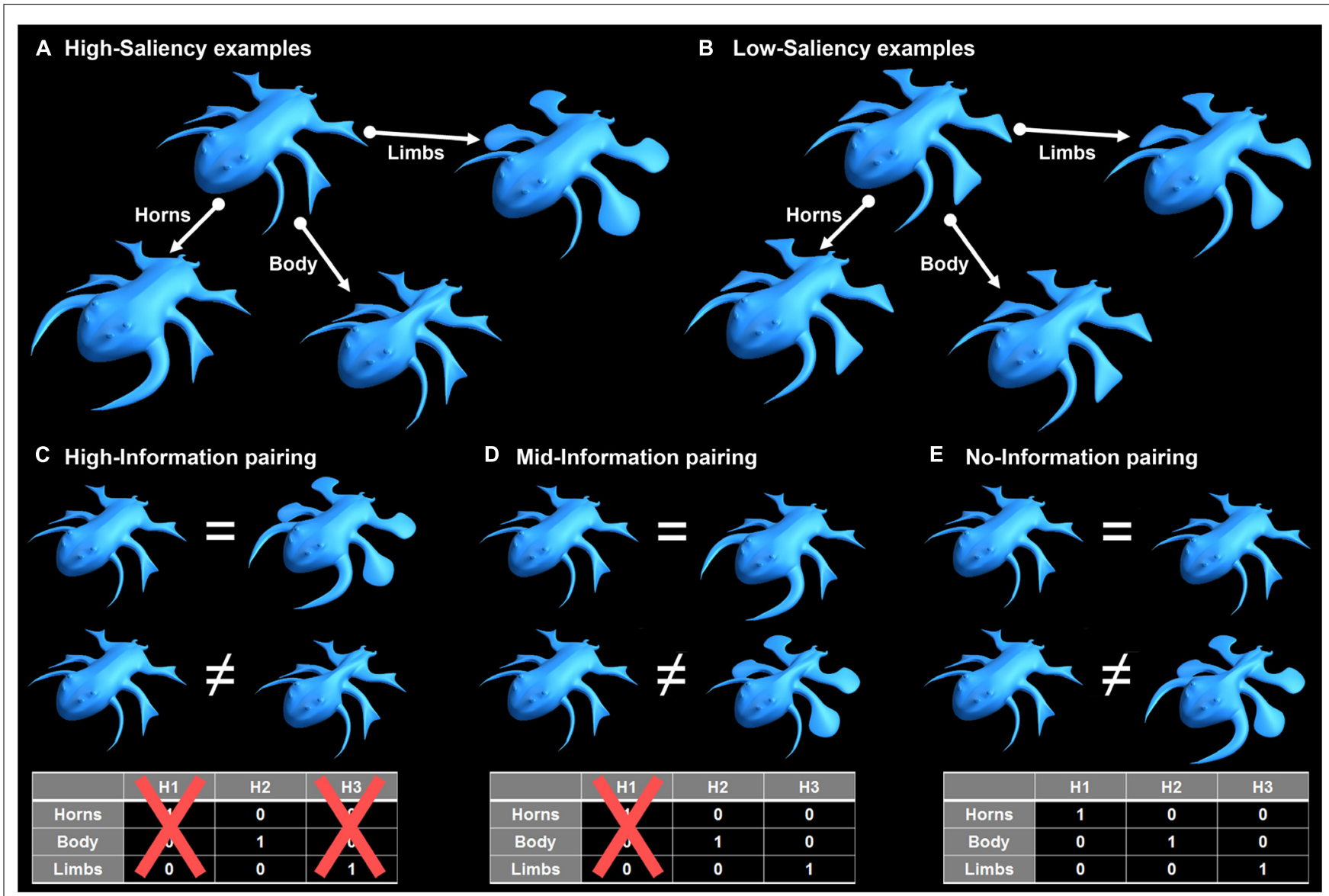

FIGURE 4 | Creature-pairing compositions. (A) Example of high-saliency feature dimensions. (B) Example of low-saliency feature dimensions. (C) Examples of same-category (upper) and different-categories (lower) high-information pairs (using the high-saliency stimuli). The portion of feature dimensions detected as relevant based on a given trial determined the quantity of information provided in this trial. Here, the only feasible 'hypothesis' that could be considered by the participant was that the creatures' body width is relevant for categorization $\left(-\log _{2} 1 / 3=1.585\right.$ bits). (D) Examples of same-category (upper) and different-categories (lower) mid-information pairs. Based on these examples, either the creatures' body width or their limbs could be relevant for categorization

( $-\log _{2} 2 / 3=0.585$ bits). (E) Examples of same-category (upper) and different-categories (lower) pairs with no-information (control task). Based on these examples, the creatures' body width, their limbs or their horns could be relevant for categorization ( $-\log _{2} 3 / 3=0$ bits). In learning-blocks, the category relation between the paired stimuli could be deduced from the feedback that followed a categorization decision. The tables at the bottom describe the possible hypothesis space. When all feature dimensions are salient and participants are informed that only one feature dimension is task-relevant, VCL requires the participant deciding which of the 3 hypotheses $(\mathrm{H} 1-\mathrm{H} 3)$ is correct. When features are not salient, and the participant is more likely to be unaware of any between-stimuli differences, VCL require the participant rejecting the null hypothesis stating, "all creatures are the same". was impossible: $(A \cup B \cup C \rightarrow X) \cap(A \cup B \cup C \rightarrow X) \cap(A \cup B \cup C \rightarrow$ $X) \cap(A \cup B \cup C \rightarrow X) \cdots$ (see Schulz et al., 2007 and Shafto et al., 2008 for a similar formulation of ambiguity in induction tasks).

In the unsupervised 'learning-blocks' composition of paired stimuli was the same as in the test blocks, where paired stimuli always differed in two feature dimensions. In each test-block trial, paired creatures always differed in two feature dimensions. Samecategory pairs differed in the two irrelevant feature dimensions and were identical in the relevant one (such as in the upper pair in Figure 4C). Different-categories pairs differed in the relevant feature dimension and in one of the irrelevant feature dimensions, and were identical in the other irrelevant feature dimension (such as in the lower pair in Figure 4D). This prevented participants from making same/different categorization decision based on overall similarity between stimuli, and it ensured that all pair-wise correlations between the three feature dimensions are identical (the relevant feature dimension could not be inferred from the stimuli presentation statistics).

\section{PERFORMANCE MEASUREMENTS}

We define a "Hit" as correctly identifying two creatures as members of the same-category, and a "False-Alarm" as incorrectly identifying two creatures of different-categories as members of the samecategory. Based on the Hit and False-Alarm rate we calculated the participant's accuracy using the non-parametric measure Aprime (Grier, 1971; Stanislaw and Todorov, 1999). A-prime $=0.5$ indicates chance-level performance and A-prime $=1.0$ indicates perfect performance. A-prime scores close to 0.0 indicate high sensitivity to category identity but with consistently reversed 
responses (i.e., categorizing items from two categories as belonging to the same category, and categorizing items from the same category as belonging to different categories). Hit rate and False-Alarm rate are defined as:

$$
\begin{gathered}
H=\text { Hit rate }=\frac{\text { Hits }}{\text { Hits }+ \text { Misses }} \\
F=\text { False Alarm rate }=\frac{\text { False Alarms }}{\text { False Alarms }+ \text { Correct Rejections }}
\end{gathered}
$$

A-prime is defined as:

$$
A^{\prime}=0.5+\operatorname{sign}(H-F) \times \frac{(H-F)^{2}+|H-F|}{4 \times \max (H, F)-4 \times H \times F}
$$

\section{KEY PERFORMANCE BENCHMARKS}

Participants' learning capabilities were evaluated based on several benchmarks accounting for the constraints of stimuli pairing in learning and test blocks, and the correlations between feature dimensions: making random same/different decisions would yield chance performance, A-prime $=0.5$; categorizing based on the relevant feature dimension would yield perfect performance, A-prime $=1$; systematically referring to an irrelevant feature dimension during a test block or during an unsupervised 'learningblock' would yield an A-prime $=0.12$ (with Hit rate $=0$ and False-Alarm rate $=0.5$ ); systematically referring to an irrelevant feature dimension during a learning block with mid-information feedback would yield an A-prime $=0.5$; due to the anti-correlation between the task-relevant feature dimension and the irrelevant feature dimensions, in high-information learning-blocks, systematically referring to an irrelevant feature dimension would yield an A-prime $=0$ (see Appendix B, in the online supplemental, for data exclusion criteria).

\section{RESULTS}

\section{OVERVIEW}

There are two main objectives for the analysis: (i) Showing that the negative impact of ambiguous feedback on VCL is context dependent, most likely to be manifested in low-saliency conditions; (ii) Showing that participants' performances in both low-saliency VCL tasks (mid-information and high-information) depended more on perceptual learning than their performances in the corresponding high-saliency VCL tasks.

\section{PRE-LEARNING PERFORMANCE}

A two-way ANOVA with feature saliency and feedback information as independent variables, and categorization accuracy (A-prime) in the pre-learning test block (T1) as the dependent variable shows no significant interaction between feature saliency and feedback information prior to learning $F(1,92)=1.48, p=0.23$, no significant feedback information main effect $F(1,92)=0.39$, and no feature saliency main effect $F(1,92)=0.74$. This analysis confirms that the initial mean categorization accuracy in the four experimental conditions was the same (Figure 5A). A two-way ANOVA with feature saliency and feedback information as independent variables, and Hit rate in the pre-learning test block (T1) as the dependent variable shows, as well, no significant interaction between feature saliency and feedback information, $F(1,92)=2.35, p=0.13$, no significant feedback information main effect, $F(1,92)=1.29, p=0.26$, and no feature saliency main effect $F(1,92)=1.52, p=0.22$.

Differences between experimental conditions in pre-learning categorization patterns were evident in False-Alarm rates (the participants' tendency to incorrectly identify two creatures as belonging to the same category). A two-way ANOVA with feature saliency and feedback information as independent variables, and participants' False-Alarm rate in the pre-learning test block (T1) as the dependent variable, shows no significant interaction between feature saliency and feedback information prior to learning $F(1,92)=0.35$, no significant feedback information main effect $F(1,92)=0.19$, but a significant feature saliency main effect $F(1,92)=28.40, p<0.00001, \eta_{p}^{2}=0.24$. Specifically, the initial False-Alarm rate was greater in the low-saliency conditions (Mean $=0.61 \pm \mathrm{SD}=0.20$ ), where between stimuli differences were harder to detect, than in the high-saliency conditions $(0.36 \pm 0.24)$. This difference in False-Alarm rate was

\section{A Pre-learning accuracy [T1]}

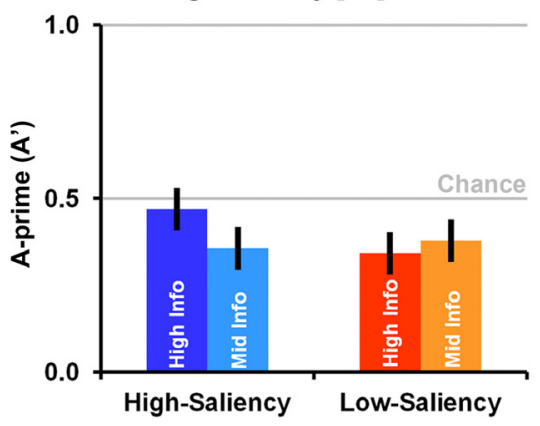

\section{B Pre-learning ROC (High-Saliency)}

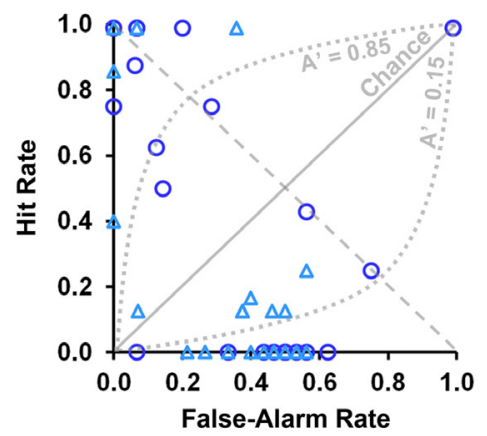

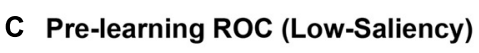

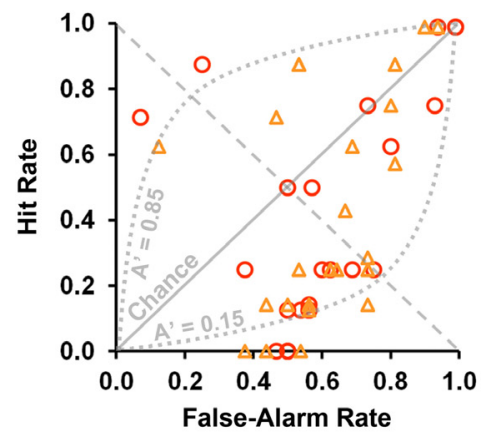

FIGURE 5 | (A) Mean (error bars are SE of the mean) pre-learning A-prime scores in the high-information and mid-information feedback conditions (high-saliency vs. low-saliency). (B) Receiver Operation Characteristics (ROC) diagram for the high-saliency conditions (blue-purple circles $=$ high-information; light blue triangles = mid-information). Each circle/triangle represents a single case. (C) ROC diagram for the low-saliency conditions (red circles = high-information; orange triangles $=$ mid-information). 
associated with participants' response bias but had little impact on their overall categorization accuracy (A-prime score) due to corresponding differences in Hit rate (though these were statistically insignificant).

In the high-saliency conditions we find that most participants either exhibited high Hit rate and low False-Alarm rate indicating that they guessed the right feature dimension prior to the first learning-block, or near zero Hit rate and near 0.5 False-Alarm rate, which indicates participants that persistently categorized stimuli based on one of the two irrelevant feature dimensions. This suggests that in high-saliency conditions participants became quickly aware to the difference between stimuli. This is presented in Figure $\mathbf{5 B}$ as the area not confounded by the $0.85>$ A-prime $>0.15$ arches on the Receiver Operation Characteristics (ROC) diagram. A-prime scores higher than 0.85 or lower than 0.15 roughly match higher than $80 \%$ or lower than $20 \%$ correct, respectively $(80 \%$ correct indicates cases with $<6$ errors in the first test-block). These indicate cases where a participant rapidly detected differences in one of the three feature dimensions in which stimuli varied. In the low-saliency conditions, on the other hand, many participants had both high Hit rate and high False-Alarm rate, where the vast majority of participants had closer to chance level performance (Figure 5C). That is, in the pre-learning phase, in low-saliency conditions, participants were much more likely to perceive paired creatures as belonging to the same-category, presumably due to not yet detecting the low-saliency differences in either the relevant or irrelevant feature dimensions.

Table 1 shows the number of participants who exhibited high persistency in categorizing stimuli based on a specific feature dimension in the pre-learning phase, in each condition. A Fisher exact test shows that the portion of cases exhibiting high persistency in categorizing stimuli based on a specific feature dimension in the high-saliency conditions (28/48) was significantly higher (more than three times higher) than in the low-saliency conditions (9/48), $p=0.0001$ (two-tailed). There were no significant differences in performance pattern between the mid-information and high-information conditions

Table 1 | Number of participants who showed accuracy levels of A-prime $>0.85$ or A-prime $<0.15$ in the pre-learning stage $(24$ participants in each condition).

\begin{tabular}{cllll}
\hline \multicolumn{2}{c}{ Condition } & $\mathbf{A}^{\prime}>\mathbf{0 . 8 5}$ & $\mathbf{A}^{\prime}<\mathbf{0 . 1 5}$ & $\begin{array}{l}\text { High } \\
\text { persistency }\end{array}$ \\
\hline High-saliency & High-info & $6 / 24$ & $9 / 24$ & \\
& Mid-info & $5 / 24$ & $8 / 24$ & \\
& Total & $11 / 48$ & $17 / 48$ & $28 / 48$ \\
Low-saliency & High-info & $2 / 24$ & $4 / 24$ & \\
& Mid-info & $0 / 24$ & $3 / 24$ & \\
& Total & $2 / 48$ & $7 / 48$ & $9 / 48$ \\
\hline
\end{tabular}

These A-prime values indicate participants that were highly persistent in categorizing creatures based on the predetermined feature dimension or one of the two irrelevant feature dimensions, respectively. An A-prime score of 0.85 roughly matches $d$-prime $\left(d^{\prime}\right)=1.5$, and is at about $80 \%$ correct. in either feature saliency level, both $p>0.4$ (two-tailed, Fisher exact).

\section{INTERACTIVE IMPACT OF FEEDBACK INFORMATION AND FEATURE SALIENCY ON VCL DYNAMICS}

We first compared the learning trajectories from the four conditions by accounting to the mean performances (A-prime scores) in the test and learning blocks combined (T1, L1, T2, L2, T3, L3, T4). A three-way ANOVA with feature saliency (low-saliency vs. high-saliency) and feedback information (mid-information vs. high-information) as independent between-subjects variables, test/learning-phase (T1, L1, T2, L2, T3, L3, T4) as a repeated measure variable, and performance (A-prime) as the dependent variable, shows a three-way interaction evident as a significant feature saliency by feedback information by learning-phase linear contrast (contrasting between the learning trajectories of the four conditions, assuming a simple linear model), $F(1,92)=3.77$, $p=0.055, \eta_{\mathrm{p}}^{2}=0.04$. Additionally, this ANOVA shows a significant main effect of feature-saliency, $F(1,92)=10.23, p<0.002$, $\eta_{\mathrm{p}}^{2}=0.10$ (high-saliency $>$ low-saliency), and a trend toward a significant main effect of feedback information, $F(1,92)=3.67$, $p=0.059, \eta_{p}^{2}=0.04$ (high-information $>$ mid-information). This ANOVA also shows that differences between the learning trajectories of the two feature saliency conditions are best explained by a cubic contrast, $F(1,92)=10.42, p<0.002, \eta_{\mathrm{p}}^{2}=0.10$, indicating that differences between saliency conditions were maximized in the second test block (T2; unlike a linear contrast which indicates a monotonic change in differences between conditions as the VCL task progresses). There were no significant polynomial contrasts between the learning trajectories of the mid-information and high-information feedback conditions, all $p>0.15$.

Post hoc analyses show that the above three-way interaction primarily results from slower learning and overall lower categorization performances in the low-saliency mid-information condition. A two-way ANOVA comparing the two low-saliency conditions, with feedback information as an independent variables, learning-phase (T1, L1, T2, L2, T3, L3, T4) as a repeated measure variable, and performance (A-prime) as the dependent variable, shows a significant linear contrast between the mid- and high-information feedback conditions, $F(1,46)=5.28$, $p<0.03, \eta_{\mathrm{p}}^{2}=0.10$, a trend towards a significant feedback information simple main effect when accounting to all blocks, $F(1,46)=3.41, p=0.071, \eta_{p}^{2}=0.07$, and a significant feedback information simple main effect when accounting only to the blocks starting from T2 (following the first learning block), $F(1,46)=4.84, p<0.04, \eta_{p}^{2}=0.09$. This indicates faster learning (steeper ascending slope) and overall better performance in the low-saliency high-information condition than in the lowsaliency mid-information condition (Figure 6B). A two-way ANOVA compering the two mid-information conditions shows that mean performance in the low-saliency mid-information condition was lower than in the high-saliency mid-information condition. This was evident as a significant quadratic contrast, $F(1,46)=8.15, p<0.007, \eta_{\mathrm{p}}^{2}=0.15$, and a significant feature saliency simple main effect, $F(1,46)=6.35, p<0.02$, $\eta_{\mathrm{p}}^{2}=0.12$ 
A Learning trajectories (High-Saliency)

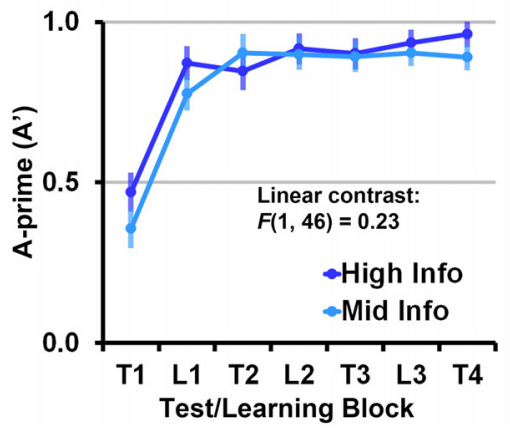

B Learning trajectories (Low-Saliency)

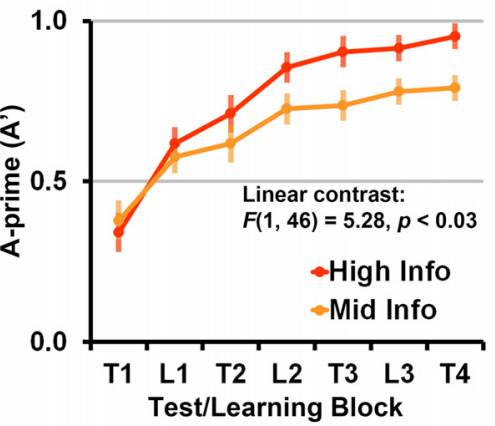

C Post-learning accuracy [T2, T3 \& T4]

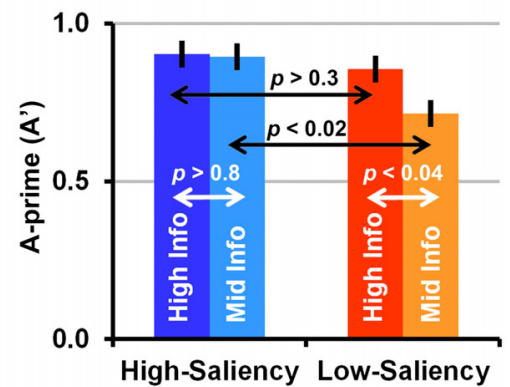

FIGURE 6 | (A) Mean (error bars are SE of the mean) A-prime scores in all blocks (T1, L1, T2, L2, T3, L3, T4) in the high-saliency conditions. (B) Mean A-prime scores in all blocks in the low-saliency conditions. (C) Mean post-learning A-prime scores (mean of T2, T3, and T4) in the high-information and mid-information feedback conditions (high-saliency vs. low-saliency).
In the high-saliency conditions there was no difference between the mid-information and high-information feedback conditions, $F(1,46)=0.67$, indicating that learning in high-saliency conditions was not significantly affected by the feedback ambiguity manipulation (Figure 6A). Importantly, we found that learning in the high-saliency high-information condition was better than in the low-saliency high-information condition. This was evident as a significant linear contrast, $F(1,46)=6.34, p<0.02$, $\eta_{\mathrm{p}}^{2}=0.12$, and a significant feature saliency simple main effect, $F(1,46)=3.88, p=0.055, \eta_{\mathrm{p}}^{2}=0.08$, indicating faster learning and overall better performance in the high-information highsaliency condition than in the high-information low-saliency condition. That is, perceptual learning played a significant role in both low-saliency conditions. Nevertheless, unlike the lowsaliency mid-information condition where learning was fairly impaired throughout the entire task, in the low-saliency highinformation condition later-phase performances matched the performances observed in the two high-saliency conditions (see also Figure 7).

The following analysis is based only on the mean performances in the four test blocks, where the composition of trials in the two feedback information conditions (for each given feature saliency level) was identical. This analysis shows that the exceptionally lower performance in the low-saliency mid-information VCL condition was evident as lower mean performance in all postlearning test trials (T2, T3, T4). A three-way ANOVA with feature saliency (low-saliency vs. high-saliency) and feedback information (mid-information vs. high-information) as independent variables, learning-phase (pre-learning [T1] versus post-learning [T2, T3, T4]) as a repeated measure variable, and categorization accuracy (A-prime) as the dependent variable, shows a significant three-way interaction between feature-saliency, feedback information, and learning-phase, $F(1,92)=4.29, p<0.05, \eta_{\mathrm{p}}^{2}=0.045$ (Greenhouse-Geisser corrected).

Post hoc analyses show that the post-learning mean performance in the low-saliency mid-information condition was lower than all other conditions, whereas the mean performance in the three other conditions was largely matched (Figure 6C). When contrasting between the two high-saliency conditions we found no simple main effect of feedback information, $F(1,46)=0.02$, but we did find a simple main effect of feedback information when contrasting the two low-saliency conditions, $F(1,46)=4.84$,
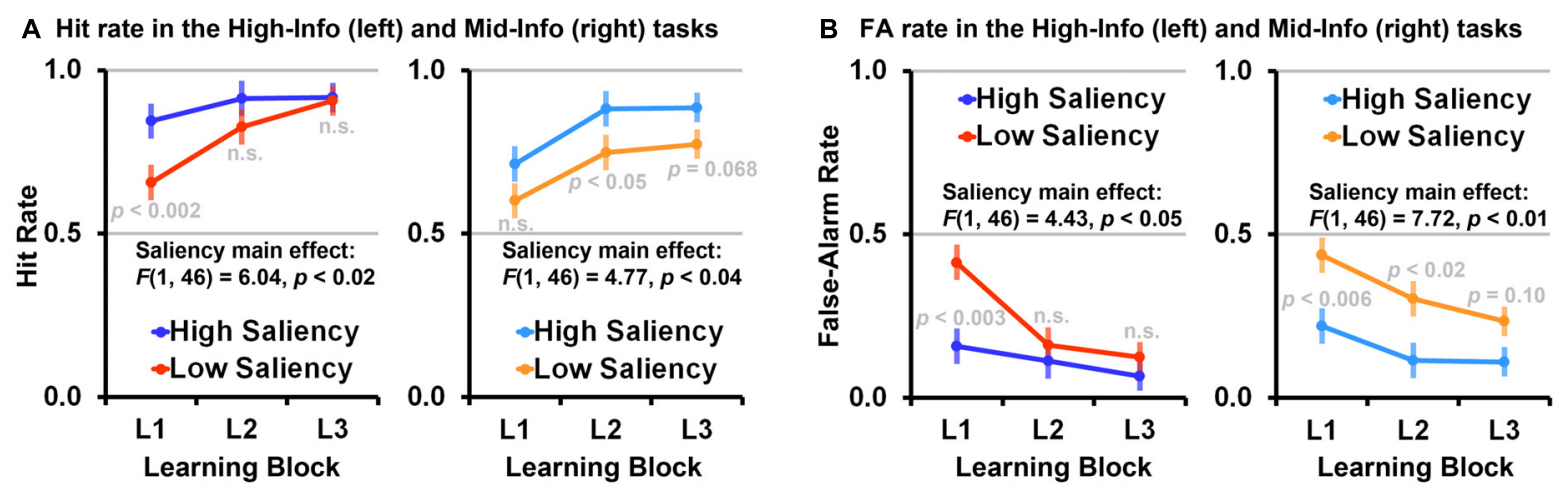

FIGURE 7 | (A) Differences in mean (error bars are SE of the mean) Hit rates in the learning blocks (L1, L2, L3) attributed to the saliency manipulation, in the high-information (left) and mid-information (right) conditions. (B) Differences in mean False-Alarm rates (n.s. indicates $p>0.10$ ). 
$p<0.04, \eta_{\mathrm{p}}^{2}=0.09$. When contrasting between the two high-information conditions we found no significant simple main effects of feature saliency, $F(1,46)=0.95$, but we found a simple main effects of feature saliency when contrasting the two midinformation conditions $F(1,46)=6.79, p<0.02, \eta_{\mathrm{p}}^{2}=0.13$. Post-learning performances in all four conditions were above chance (A-prime $=0.5$ ), all $p<0.005$. There were no significant pre-learning (T1) simple main effects, all $p>0.15$ (Figure 5A).

\section{PERCEPTUAL LEARNING}

To further investigate the role of perceptual learning in lowsaliency VCL, we looked at the impact of the feature saliency manipulation on the participants' Hit rate and False-Alarm rate in the learning blocks of each feedback information condition, taking advantage of the composition of these trials. Specifically, in high-information learning trials the task-relevant feature dimension was the only feature dimension discriminating between different-categories paired creatures, and thus performance in these trials is less affected by having several features with potential discriminative value competing for attention.

A three-way ANOVA with feature saliency (low-saliency vs. high-saliency) and feedback information (mid-information vs. high-information) as independent between-subjects variables, learning-phase (L1, L2, L3) as a repeated measure variable, and Hit rate as the dependent variable, shows a trend toward a significant feature saliency by feedback information by learningphase linear contrast, $F(1,92)=3.19, p=0.077, \eta_{\mathrm{p}}^{2}=0.03$, a significant main effect of feature saliency, $F(1,92)=10.26$, $p<0.002, \eta_{\mathrm{p}}^{2}=0.10$ (high-saliency $>$ low-saliency), and a significant main effect of feedback information, $F(1,92)=5.26$, $p<0.03, \eta_{\mathrm{p}}^{2}=0.05$ (high-information $>$ mid-information) This ANOVA also shows differences between the learning trajectories of the two feature saliency conditions evident as a trend toward a significant linear contrast, $F(1,92)=3.36$, $p=0.07, \eta_{\mathrm{p}}^{2}=0.03$. There were no significant polynomial contrasts between the learning trajectories of the midinformation and high-information feedback conditions, all $p>0.35$. Largely, this ANOVA mirrors the effects observed in the above analysis with A-prime as the dependent variable (Figure 7A).

A three-way ANOVA with feature saliency (low-saliency vs. high-saliency) and feedback information (mid-information vs. high-information) as independent between-subjects variables, learning-phase (L1, L2, L3) as a repeated measure variable, and False-Alarm rate as the dependent variable, shows a trend toward a significant feature saliency by feedback information by learningphase linear contrast, $F(1,92)=3.19, p=0.098, \eta_{\mathrm{p}}^{2}=0.03$, and a main effect of feature saliency, $F(1,92)=12.26, p<0.001$, $\eta_{\mathrm{p}}^{2}=0.12$ (low-saliency $>$ high-saliency). There was no significant feedback information main effect, $F(1,92)=2.20, p=0.14$. This ANOVA also shows a feature saliency main effect evident as a significant linear contrast, $F(1,92)=7.59, p<0.007$, $\eta_{\mathrm{p}}^{2}=0.08$ (with a steeper descending slope in the low-saliency tasks due to low False-Alarm rate in the high-saliency tasks, starting at L1; a close to floor effect). There were no significant polynomial contrasts between the learning trajectories of the mid-information and high-information feedback conditions, all $p>0.50$ (Figure 7B).

Unlike the Hit rate, which was significantly affected both by the feature saliency and feedback information manipulations, the False-Alarm rate was primarily affected by the feature saliency manipulation with substantially higher False-Alarm rate in low saliency tasks. This indicates, as might be expected, that lowsaliency primarily (yet not exclusively) compromise VCL by reducing the capacity to differentiate between stimuli from two distinct categories.

\section{CATEGORIZATION PERFORMANCE IN THE CONTROL TASKS}

A three-way ANOVA with feature saliency (low-saliency vs. high-saliency) and feedback-availability (no-information feedback vs. unsupervised categorization with no feedback) as independent variables, learning-phase [pre-learning (T1) vs. post-learning (T2, T3, T4)] as a repeated measure variable, and categorization accuracy (A-prime) as the dependent variable, shows no learning main effect (pre-learning to postlearning), $F(1,93)=0.13, p>0.7$, or any other significant main effect or interaction effect, all $p>0.1$ (see Table 2). This analysis confirms that the categorization accuracies and the performance improvement observed in the four experimental conditions (mid-information and high-information) could only be attributed to feedback information, and not to cumulative experience with the stimuli. We note that in the control tasks participants had greater tendency not to respond on time, or to exhibit stereotypical persistent response pattern ("yea-sayers" or "nay-sayers"). Being relatively common, cases with such performance patterns were not excluded from this analysis.

\section{DISCUSSION}

We tested the interaction between feature saliency and feedback information in visual category learning (VCL) tasks as a mean to explore interactions between perceptual learning and attentional learning processes in different VCL scenarios (high-saliency vs. low-saliency scenarios). We found that in high-saliency tasks participants reached highest accuracies within the first learning-block, surprisingly also in the midinformation feedback VCL task where learning trial were ambiguous (Figure 6A). In the low-saliency high-information feedback condition we found that VCL required more learning trials

Table 2 | Mean ( \pm SD) A-prime in the control tasks with no-information feedback and unsupervised categorization tasks where no feedback was provided.

\begin{tabular}{llll}
\hline \multicolumn{1}{c}{ Condition } & Pre [T1] & Post [T2, T3, and T4] \\
\hline High-saliency & No-info & $0.31 \pm 0.30$ & $0.31 \pm 0.31$ \\
& Unsupervised & $0.38 \pm 0.35$ & $0.45 \pm 0.41$ \\
Low-saliency & No-info & $0.40 \pm 0.30$ & $0.39 \pm 0.37$ \\
& Unsupervised & $0.27 \pm 0.15$ & $0.23 \pm 0.23$
\end{tabular}

Here we find no pre-learning to post-learning performance changes. 
than in the high-saliency high-information condition. Nevertheless, when having high-information feedback available, participants ultimately reached the same categorization accuracies observed in the two high-saliency tasks (Figures 6B and 7A,Bleft).

Importantly, as we hypothesized, we found that ambiguous feedback substantially impaired VCL in low-saliency tasks, resulting in significantly lower categorization accuracies than those observed in the three other experimental conditions (Figure 6C). We suggest that in low-feature-saliency scenarios with ambiguous feedback, learning is more difficult due to a "chicken-or-the-egg" cognitive loop paradox. In such VCL scenarios there are two cognitive challenges that need to be resolved: (i) becoming aware to important feature-wise differences between stimuli and increasing sensitivity to these differences, (ii) and determining which feature-dimensions are relevant for categorization. The paradox is that resolving each challenge depends on resolving the other challenge first. On the one hand, to be effective, perceptual learning requires persistently directing attention to specific feature dimension (Goldstone, 1994; Gilbert et al., 2001; Kruschke, 2001); but this is unlikely to happen when there is an ambiguity regarding which feature dimension needs to be attended. On the other hand, attentional learning requires using feedback information for systematically valuing which feature dimension is important for categorization, while ignoring irrelevant feature dimensions; but attentional learning is likely to be compromised when there are only vague impressions of any differences between the perceived objects.

Challenges distinct to low-saliency VCL scenarios were already evident as differences in performance patterns between the highsaliency and low-saliency conditions prior to learning (T1): In high-saliency conditions, most participants showed high persistency in categorizing the creatures based on one of the three feature dimensions in which the creatures varied (Table 1; Figure 5B). The frequently observed A-prime $>0.85$ or $A$-prime $<0.15$ scores indicates that in high-saliency tasks, prior to learning, most participants were likely to instantly detect at least one of the three differentiating feature dimensions and to consistently use it for categorizing the creatures. We can assume that in the pre-learning phase, in high-saliency tasks, participants asked themselves "which of these differentiating attributes is most relevant for categorizing these creatures?" On the other hand, in low-saliency conditions most participants performed closer to chance level (A-prime $=0.5$ ), where many participants had both high Hit rate and high False-Alarm rate. This indicates that in low-saliency tasks participants perceived most paired creatures as being the same, presumably not detecting any differences (Table 1; Figure 5C). Here many participants may have asked themselves "do these creatures differ at all, and if yes, in what way?" These two distinct starting points impose different constraints on the cognitive strategies that participants could employ during later VCL phases.

In high-saliency tasks, in the first learning block (L1), we found that participants could use the feedback information from few trials for quickly inferring if the feature dimension by which they categorized the creatures prior to learning (T1) is the relevant feature dimension. This was true even when provided with mid-information feedback. If the feedback indicated frequent errors, attention could be quickly redirected to one of the other two salient feature dimensions by which creatures varied. In high-saliency tasks there was little or no need for perceptual learning to take place, and the primary cognitive challenge in these tasks was testing hypotheses whether one feature or the other is relevant for categorization. This enabled the rapid inference of a generalized categorization rule.

In low-saliency VCL tasks the detection of feature dimensions in which the creatures varied was initially much harder than in high-saliency tasks. Thus, it was most unlikely that during the pre-learning test block (T1) the participants would form a solid 'hypothesis space' where there are few alternative categorization rules to choose from. In the first learning block (L1), instead of using the feedback for systematically testing which feature dimension is relevant for categorization, participants primarily used the feedback for perceptual learning and for further detection of possibly other diagnostic feature dimensions. The feedback enabled cumulating evidences indicating that apparently identical paired creatures are in fact different. This forced participants to continue searching for diagnostic feature dimensions, as it also enabled them to validate if some vague perceived differences between creatures represent concrete differences in a specific feature dimension that deserve being further attended. This enabled increasing the sensitivity to this feature dimension and to reduce categorization error rate (Herzog and Fahle, 1997).

Being provided with mid-information feedback increased the odds that few vague impressions of possibly differentiating feature dimensions would be simultaneously considered as relevant for categorizing creatures. Not attending to only one low-saliency feature dimension at a time was likely to compromise perceptual learning, and thus it hindered the increase in sensitivity to partially attended feature dimensions. In turn, not becoming sufficiently sensitive to differences in a given feature dimension reduced the participants' confidence that this feature dimension has diagnostic value, further reducing the odds that it would be frequently attended, and thus further compromising perceptual learning.

It is also possible that in low-saliency tasks some participants attended a specific feature dimension of the creatures (e.g., the creatures heads) across multiple successive trials, even prior to learning (T1). If this feature was one of the three feature dimensions in which stimuli varied, this could result in the participant becoming more sensitive to between-creatures differences in this feature dimension even without receiving feedback (Petrov et al., 2006; Liu et al., 2012). If the feature dimension relevant for categorization was consistently attended during T1, such accidental direction of attention could enable faster learning. However, if one of the two irrelevant feature dimensions was consistently attended during $\mathrm{T} 1$, increased sensitivity to this feature dimension could have stunted the learning of the task-relevant feature dimension, resulting in slower VCL (Kruschke, 2003; Kruschke et al., 2005; Bott et al., 2007; Blair et al., 2009).

In low-saliency tasks, providing participants with highinformation feedback in L1 could allow participants an opportunity to confirm if the initially attended (yet still poorly represented) feature dimension is the one relevant for categorization. However, 
due to relatively poor representation, this learning was not as fast as learning in high-saliency conditions since it required either slow increase in sensitivity to the earlier detected differences, or the search for other low-saliency differences between creatures. This should have been followed by further perceptual learning for increasing sensitivity to the detected differences. If the feature dimension most attended in T1 was one of the two irrelevant feature dimensions (the more likely scenario), the introduction of mid-information feedback in L1 was likely to have an initial negative impact on VCL, reinforcing the impression that an irrelevant low-saliency feature dimension is in fact relevant for categorization. This could prevent the participant from searching for the task relevant feature dimension.

Despite the suggested cognitive loop paradox, we found that learning in the low-saliency mid-information condition was ultimately feasible, with above chance mean performance (Figure 6C). This can be explained, at least partially, by characteristics of the current experimental design. First, low-saliency was not 'too low' based on the pre-learning (T1) performances in the low-saliency tasks, it is clear that differences between paired creatures were hard to detect (harder than in the high-saliency tasks) within the short stimuli presentation time. Nevertheless, in the low-saliency, high-information condition we found that participants eventually reached performance levels matching those observed in the high-saliency conditions (though it required more learning trials, Figures 6 and 7). This suggests that the current experimental design does not fully exhaust perceptual learning capacities of normal adults.

Secondly, in the current experimental design ambiguous feedback was not 'too ambiguous' - based on performances in the high-saliency mid-information condition, it is clear that when having a total of three feature dimensions in which stimuli vary, and when in each given trial the task-relevant feature dimension is competing for the participant's attention with only one similarly salient irrelevant feature dimension, feedback ambiguity is manageable and in fact seem to have negligible evident impact on VCL performances. This is clearly not expected to be the case in VCL tasks with even more ambiguous feedback. Future studies may investigate the interaction between feature saliency and feedback information in more 'extreme' feedback ambiguity conditions. For example, if increasing the total number of varying feature dimensions to five, where in each trial the paired creatures differ in the task-relevant feature dimension and a random combination of three of the irrelevant feature dimensions, learning the categorization rule may (would) take longer even in high-saliency conditions. Moreover, in such scenarios it may become more likely that learning in low-saliency VCL tasks would be utterly compromised due to having attention being directed to the task relevant feature dimension even less frequently than in the mid-information conditions that we tested here. In such scenarios it would become even less likely that brain activation associated with task-relevant features would reach the threshold required for significant changes in representation to take place (Goldstone, 1994; Gilbert et al., 2001; Kruschke, 2001).

The reported differences in performances between the lowsaliency mid-information condition and the low-saliency highinformation condition may contribute to the ongoing debate regarding the role of attention control in perceptual learning, to which we referred in more detail in the Introduction (Ahissar and Hochstein, 1993; Schoups et al., 2001; Herzog and Fahle, 2002; Seitz et al., 2009; Kourtzi, 2010; Roelfsema et al., 2010; Aberg and Herzog, 2012). Here we show that VCL performance in the low-saliency mid-information condition, where perceptual learning had to take place under conditions that required distributing attention among few feature dimensions, was most impaired. Nevertheless our data does not allow directly testing the degree to which participants' sensitivity to the relevant and irrelevant feature dimensions has been changed following learning, apart from how these changes impacted categorization performances (which are likely to reflect changes in representation of the task-relevant feature dimension). Testing participants' sensitivity to differences between paired creatures in each feature dimension separately, before and after VCL has been completed, may indicate if perceptual learning took place, if it is restricted to the task-relevant feature dimension, or if it is evident as increased sensitivity also to task-irrelevant feature dimensions (we advise having a short prelearning sensitivity test, since extensive exposure to the stimuli may facilitate unsupervised perceptual learning to some arbitrary features in which stimuli vary).

Related to the above, we suggest that as much as ambiguous feedback may compromise VCL, it is more likely to result in an increased perceptual sensitivity to irrelevant feature dimensions. This is due to greater correlations between the task-relevant feature dimension and the irrelevant ones, which is likely to result in more frequent associations between irrelevant feature dimensions and the feedback (or reward) that follows a categorization decision. This may reinforce irrelevant feature dimensions more frequently than in higher-information feedback conditions, where only the task-relevant feature dimension is frequently reinforced. For example, when ' $A$ ' denotes the relevant feature-dimension, ' $B$,' ' $C$, ' $D$,' and ' $E$ ' the irrelevant feature-dimensions and ' $X$ ' the categorization decision outcome, in a scenario with trials composition such as $(A \cup B \rightarrow X) \cap(A \cup D \rightarrow X) \cap(A \cup C \rightarrow X) \cap(A \cup E \rightarrow$ $X) \cap(A \cup D \rightarrow X) \cdots$ there would be relatively sparse association between each of the irrelevant feature-dimensions and the decision outcome ( $25 \%$ of the trials; $-\log _{2} 2 / 5=1.322$ bits of information per trial). On the other hand, in a scenario with trial composition such as $(A \cup B \cup C \cup D \rightarrow X) \cap(A \cup C \cup D \cup E \rightarrow$ $X) \cap(A \cup B \cup C \cup D \rightarrow X) \cap(A \cup B \cup C \cup E \rightarrow X) \cap(A \cup B \cup D \cup E \rightarrow$ $X) \cdots\left(75 \%\right.$ of the trials; $-\log _{2} 4 / 5=0.322$ bits per trial) it would become more likely that at least some of the task-irrelevant feature-dimensions would be reinforced following VCL.

That is, the administration of learning trials and their informativeness may greatly impact the effectiveness of VCL and, the odds that VCL would result in evident changes in neural representation of task-irrelevant feature dimensions (see Jiang et al., 2007; Folstein et al., 2013, 2014, for related findings). More generally, we predict that in order to observe VCL associated with an increased sensitivity to irrelevant feature dimensions, the VCL task has to meet two requirements: (i) the number of irrelevant feature dimensions should be small, and (ii) assuming a given small number of irrelevant feature dimensions, the feedback information in VCL tasks should be minimized so to increase the odds that attention would be distributed between these few irrelevant 
feature dimensions, so that at least some of these feature dimensions would be frequently reinforced (see Watanabe and Sasaki, 2015, for a related discussion).

Finally, future studies may also investigate how VCL, under different feature saliency conditions, can be optimized while accounting for the fact that when categorizing same-category stimuli, the information gained is not with the same use as the information gained when categorizing different-category stimuli (even when receiving the same information quantity). Specifically, informative same-category stimuli comparison is most effective for highlighting the permitted within category variability, whereas informative different-categories stimuli comparison highlights between categories differences (Hammer et al., 2009a,b, 2010; Kurtz et al., 2013; Carvalho and Goldstone, 2014). For this reason, in high-saliency VCL tasks comparison of same-category examples would be valuable for learning which feature dimensions should be ignored (contributing to attentional learning). On the other hand, comparison of same-category examples would not have much value in low-saliency VCL tasks where the main challenge is to detect important subtle differences between stimuli and to become more sensitive to these differences (requiring perceptual learning). In such scenarios, informative comparison of different-categories exemplars would be most critical to VCL. These ideas have implications for understanding possible differences between general neurocognitive mechanisms of VCL, versus those that are specifically critical for acquiring expertise (Scott et al., 2008).

In sum, the current study, despite its limitations resulting from not exploring a broader range of feature saliency and feedback ambiguity, provides a methodological and theoretical framework that if adopted may contribute to the understanding of how neurocognitive mechanisms of perception, attention, reasoning and learning are jointly being used, or failed to be used, in varying VCL scenarios.

\section{ACKNOWLEDGMENTS}

This study was supported by the Stanford neuro-innovate grant for innovative research in vision science to Rubi Hammer, the NSF (BCS-0720135) and NIH (R01HD056105) grants to Vladimir Sloutsky, and NIH (R01EY019279-01A1) grant to Kalanit GrillSpector. We thank Jim Kloet (Northwestern University) for his helpful comments on this manuscript. Part of the findings reported here was published as a conference paper (Hammer et al., 2012).

\section{SUPPLEMENTARY MATERIAL}

The Supplementary Material for this article can be found online at: http://www.frontiersin.org/journal/10.3389/fpsyg.2015.00074/ abstract

\section{REFERENCES}

Aberg, K. C., and Herzog, M. H. (2012). Different types of feedback change decision criterion and sensitivity differently in perceptual learning. J. Vis. 12, 3. doi: $10.1167 / 12.3 .3$

Ahissar, M., and Hochstein, S. (1993). Attentional control of early perceptual learning. Proc. Natl. Acad. Sci. U.S.A. 90, 5718-5722. doi: 10.1073/pnas.90.12.5718

Ahissar, M., and Hochstein, S. (1997). Task difficulty and the specificity of perceptual learning. Nature 387, 401-406. doi: 10.1038/387401a0
Antzoulatos, E. G., and Miller, E. K. (2011). Differences between neural activity in prefrontal cortex and striatum during learning of novel abstract categories. Neuron 71, 243-249. doi: 10.1016/j.neuron.2011.05.040

Awh, E., Belopolsky, A. V., and Theeuwes, J. (2012). Top-down versus bottom-up attentional control: a failed theoretical dichotomy. Trends Cogn. Sci. 16, 437-443. doi: 10.1016/j.tics.2012.06.010

Awh, E., and Jonides, J. (2001). Overlapping mechanisms of attention and spatial working memory. Trends Cogn. Sci. 5, 119-126. doi: 10.1016/S13646613(00)01593-X

Baluch, F., and Itti, L. (2011). Mechanisms of top-down attention. Trends Neurosci. 34, 210-224. doi: 10.1016/j.tins.2011.02.003

Blair, M. R., Watson, M. R., Walshe, R. C., and Maj, F. (2009). Extremely selective attention: eye-tracking studies of the dynamic allocation of attention to stimulus features in categorization. J. Exp. Psychol. Learn. Mem. Cogn. 35, 1196. doi: 10.1037/a0016272

Borji, A., and Itti, L. (2013). State-of-the-art in visual attention modeling. IEEE Trans. Pattern Anal. Mach. Intell. 35, 185-207. doi: 10.1109/TPAMI. 2012.89

Bott, L., Hoffman, A. B., and Murphy, G. L. (2007). Blocking in category learning. J. Exp. Psychol. Gen. 136, 685. doi: 10.1037/0096-3445.136.4.685

Carvalho, P. F., and Goldstone, R. L. (2014). Effects of interleaved and blocked study on delayed test of category learning generalization. Front. Psychol. 5:936. doi: 10.3389/fpsyg.2014.00936

Chen, L., Meier, K. M., Blair, M. R., Watson, M. R., and Wood, M. J. (2013). Temporal characteristics of overt attentional behavior during category learning. Atten. Percept. Psychophys. 75, 244-256. doi: 10.3758/s13414-012-0395-8

Chin-Parker, S., and Ross, B. H. (2004). Diagnosticity and prototypicality in category learning: a comparison of inference learning and classification learning. J. Exp. Psychol. Learn. Mem. Cogn. 30, 216. doi: 10.1037/0278-7393.30.1.216

Corbetta, M., Patel, G., and Shulman, G. L. (2008). The reorienting system of the human brain: from environment to theory of mind. Neuron 58, 306-324. doi: 10.1016/j.neuron.2008.04.017

Daniel, R., and Pollmann, S. (2010). Comparing the neural basis of monetary reward and cognitive feedback during information-integration category learning. J. Neurosci. 30, 47-55. doi: 10.1523/JNEUROSCI.2205-09.2010

Davis, T., Love, B. C., and Preston, A. R. (2012). Striatal and hippocampal entropy and recognition signals in category learning: simultaneous processes revealed by model-based fMRI. J. Exp. Psychol. Learn. Mem. Cogn. 38, 821. doi: 10.1037/a0027865

Diesendruck, G., Hammer, R., and Catz, O. (2003). Mapping the similarity space of children and adults' artifact categories. Cogn. Dev. 18, 217-231. doi: 10.1016/S0885-2014(03)00021-2

Fahle, M., and Poggio, T. (eds ). (2002). Perceptual Learning. Cambridge, MA: MIT Press.

Folstein, J. R., Palmeri, T. J., and Gauthier, I. (2013). Category learning increases discriminability of relevant object dimensions in visual cortex. Cereb. Cortex 23, 814-823. doi: 10.1093/cercor/bhs067

Folstein, J. R., Palmeri, T. J., and Gauthier, I. (2014). Perceptual advantage for category-relevant perceptual dimensions: the case of shape and motion. Front. Psychol. 5:1394. doi: 10.3389/fpsyg.2014.01394

Gazzaley, A., and Nobre, A. C. (2012). Top-down modulation: bridging selective attention and working memory. Trends Cogn. Sci. 16, 129-135. doi: 10.1016/j.tics.2011.11.014

Gilbert, C. D., Sigman, M., and Crist, R. E. (2001). The neural basis of perceptual learning. Neuron 31, 681-697. doi: 10.1016/S0896-6273(01)00424-X

Goldstone, R. L. (1994). Influences of categorization on perceptual discrimination. J. Exp. Psychol. Gen. 123, 178-200. doi: 10.1037/0096-3445.123.2.178

Goldstone, R. L. (1998). Perceptual learning. Annu. Rev. Psychol. 49, 585-612. doi: 10.1146/annurev.psych.49.1.585

Goldstone, R. L., Lippa, Y., and Shiffrin, R. M. (2001). Altering object representations through category learning. Cognition 78, 27-43. doi: 10.1016/S00100277(00)00099-8

Goldstone, R. L., and Steyvers, M. (2001). The sensitization and differentiation of dimensions during category learning. J. Exp. Psychol. Gen. 130, 116. doi: 10.1037/0096-3445.130.1.116

Greenhouse, S. W., and Geisser, S. (1959). On methods in the analysis of profile data. Psychometrika 24, 95-112. doi: 10.1007/BF02289823

Grier, J. B. (1971). Nonparametric indexes for sensitivity and bias: computing formulas. Psychol. Bull. 75, 424. doi: 10.1037/h0031246 
Hammer, R., Bar-Hillel, A., Hertz, T., Weinshall, D., and Hochstein, S. (2008). Comparison processes in category learning: from theory to behavior. Brain Res. 1225, 102-118. doi: 10.1016/j.brainres.2008.04.079

Hammer, R., Brechmann, A., Ohl, F., Weinshall, D., and Hochstein, S. (2010). Differential category learning processes: the neural basis of comparison-based learning and induction. Neuroimage 52, 699-709. doi: 10.1016/j.neuroimage.2010. 03.080

Hammer, R., and Diesendruck, G. (2005). The role of dimensional distinctiveness in children's and adults' artifact categorization. Psychol. Sci. 16, 137-144. doi: 10.1111/j.0956-7976.2005.00794.x

Hammer, R., Diesendruck, G., Weinshall, D., and Hochstein, S. (2009a). The development of category learning strategies: what makes the difference? Cognition 112, 105-119. doi: 10.1016/j.cognition.2009.03.012

Hammer, R., Hertz, T., Hochstein, S., and Weinshall, D. (2009b). Category learning from equivalence constraints. Cogn. Process. 10, 211-232. doi: 10.1007/s10339008-0243-x

Hammer, R., Hertz, T., Hochstein, S., and Weinshall, D. (2007). "Classification with positive and negative equivalence constraints: theory, computation and human experiments," in Advances in Brain, Vision, and Artificial Intelligence, Lecture Note in Computer Science, eds F. Mele, G. Ramella, S. Santillo and F. Ventriglia (Berlin Heidelberg: Springer-Verlag Press), 264-276.

Hammer, R., Sloutsky, V., and Grill-Spector, K. (2012). "The interplay between feature saliency and feedback information in visual category learning tasks," Proceedings to the 34th Annual Conference of the Cognitive Science Society, Sapporo.

Herzog, M. H., and Fahle, M. (1997). The role of feedback in learning a vernier discrimination task. Vision Res. 37, 2133-2141. doi: 10.1016/S0042 6989(97)00043-6

Herzog, M. H., and Fahle, M. (2002). Effects of grouping in contextual modulation. Nature 415, 433-436. doi: 10.1038/415433a

Hoffman, A. B., and Rehder, B. (2010). The costs of supervised classification: the effect of learning task on conceptual flexibility. J. Exp. Psychol. Gen. 139, 319. doi: $10.1037 / \mathrm{a} 0019042$

Itti, L., Koch, C., and Niebur, E. (1998). A model of saliency-based visual attention for rapid scene analysis. IEEE Trans. Pattern Anal. Mach. Intell. 20, 1254-1259. doi: $10.1109 / 34.730558$

Jiang, X., Bradley, E., Rini, R. A., Zeffiro, T., VanMeter, J., and Riesenhuber, M. (2007). Categorization training results in shape-and category-selective human neural plasticity. Neuron 53, 891-903. doi: 10.1016/j.neuron.2007. 02.015

Koch, C., and Tsuchiya, N. (2007). Attention and consciousness: two distinct brain processes. Trends Cogn. Sci. 11, 16-22. doi: 10.1016/j.tics.2006.10.012

Kourtzi, Z. (2010). Visual learning for perceptual and categorical decisions in the human brain. Vision Res. 50, 433-440. doi: 10.1016/j.visres.2009.09.025

Kruschke, J. K. (2001). Toward a unified model of attention in associative learning. J. Math. Psychol. 45, 812-863. doi: 10.1006/jmps.2000.1354

Kruschke, J. K. (2003). Attention in learning. Curr. Dir. Psychol. Sci. 12, 171-175 doi: 10.1111/1467-8721.01254

Kruschke, J. K., and Blair, N. J. (2000). Blocking and backward blocking involve learned inattention. Psychon. Bull. Rev. 7, 636-645. doi: 10.3758/BF03213001

Kruschke, J. K., Kappenman, E. S., and Hetrick, W. P. (2005). Eye gaze and individual differences consistent with learned attention in associative blocking and highlighting. J. Exp. Psychol. Learn. Mem. Cogn. 31, 830. doi: 10.1037/0278-7393.31.5.830

Kurtz, K. J., Boukrina, O., and Gentner, D. (2013). Comparison promotes learning and transfer of relational categories. J. Exp. Psychol. Learn. Mem. Cogn. 39, 1303 doi: $10.1037 / \mathrm{a} 0031847$

LaBar, K. S., Gitelman, D. R., Parrish, T. B., and Mesulam, M. (1999). Neuroanatomic overlap of working memory and spatial attention networks: a functional MRI comparison within subjects. Neuroimage 10, 695-704. doi: 10.1006/nimg.1999.0503

Liu, J., Lu, Z. L., and Dosher, B. A. (2012). Mixed training at high and low accuracy levels leads to perceptual learning without feedback. Vision Res. 61, 15-24. doi: 10.1016/j.visres.2011.12.002

Lopez-Paniagua, D., and Seger, C. A. (2011). Interactions within and between corticostriatal loops during component processes of category learning. J. Cogn. Neurosci. 23, 3068-3083. doi: 10.1162/jocn_a_00008

Love, B. C. (2002). Comparing supervised and unsupervised category learning. Psychon. Bull. Rev. 9, 829-835. doi: 10.3758/BF03196342
Lupyan, G., Rakison, D. H., and McClelland, J. L. (2007). Language is not just for talking redundant labels facilitate learning of novel categories. Psychol. Sci. 18, 1077-1083. doi: 10.1111/j.1467-9280.2007.02028.x

Maddox, W. T., Ashby, F. G., and Bohil, C. J. (2003). Delayed feedback effects on rule-based and information-integration category learning. J. Exp. Psychol. Learn. Mem. Cogn. 29, 650. doi: 10.1037/0278-7393.29.4.650

Mathy, F., Haladjian, H. H., Laurent, E., and Goldstone, R. L. (2013). Similaritydissimilarity competition in disjunctive classification tasks. Front. Psychol. 4:26. doi: 10.3389/fpsyg.2013.00026

McColeman, C. M., Barnes, J. I., Chen, L., Meier, K. M., Walshe, R. C., and Blair, M. R. (2014). Learning-induced changes in attentional allocation during categorization: a sizable catalog of attention change as measured by eye movements. PLoS ONE 9:e83302. doi: 10.1371/journal.pone.0083302

Namy, L. L., and Gentner, D. (2002). Making a silk purse out of two sow's ears: young children's use of comparison in category learning. J. Exp. Psychol. Gen. 131, 5-15. doi: 10.1037/0096-3445.131.1.5

Nosofsky, R. M. (1984). Choice, similarity, and the context theory of classification. J. Exp. Psychol. Learn. Mem. Cogn. 10, 104-114. doi: 10.1037/0278-7393.10.1.104

Nosofsky, R. M. (1986). Attention, similarity, and the identification-categorization relationship. J. Exp. Psychol. Gen. 115, 39-61. doi: 10.1037/0096-3445.115.1.39

Nosofsky, R. M., and Palmeri, T. J. (1996). Learning to classify integral-dimension stimuli. Psychon. Bull. Rev. 3, 222-226. doi: 10.3758/BF03212422

Petrov, A. A., Dosher, B. A., and Lu, Z. L. (2006). Perceptual learning without feedback in non-stationary contexts: data and model. Vision Res. 46, 3177-3197. doi: 10.1016/j.visres.2006.03.022

Rehder, B., and Hoffman, A. B. (2005). Thirty-something categorization results explained: selective attention, eye-tracking, and models of category learning. J. Exp. Psychol. Learn. Mem. Cogn. 31, 811-829. doi: 10.1037/0278-7393.31.5.811

Roelfsema, P. R., van Ooyen, A., and Watanabe, T. (2010). Perceptual learning rules based on reinforcers and attention. Trends Cogn. Sci. 14, 64-71. doi: 10.1016/j.tics.2009.11.005

Rosch, E., and Mervis, C. B. (1975). Family resemblances: studies in the internal structure of categories. Cogn. Psychol. 7, 573-605. doi: 10.1016/00100285(75)90024-9

Schoups, A., Vogels, R., Qian, N., and Orban, G. (2001). Practicing orientation identification improves orientation coding in V1 neurons. Nature 412, 549-553. doi: $10.1038 / 35087601$

Schulz, L. E., Bonawitz, E. B., and Griffiths, T. L. (2007). Can being scared cause tummy aches? Naive theories, ambiguous evidence, and preschoolers' causal inferences. Dev. Psychol. 43, 1124-1139. doi: 10.1037/0012-1649.43. 5.1124

Scott, L. S., Tanaka, J. W., Sheinberg, D. L., and Curran, T. (2008). The role of category learning in the acquisition and retention of perceptual expertise: a behavioral and neurophysiological study. Brain Res. 1210, 204-215. doi: 10.1016/j.brainres.2008.02.054

Seitz, A. R., Kim, D., and Watanabe, T. (2009). Rewards evoke learning of unconsciously processed visual stimuli in adult humans. Neuron 61, 700-707. doi: 10.1016/j.neuron.2009.01.016

Serences, J. T., and Yantis, S. (2006). Selective visual attention and perceptual coherence. Trends Cogn. Sci. 10, 38-45. doi: 10.1016/j.tics.2005.11.008

Shafto, P., Kemp, C., Bonawitz, E. B., Coley, J. D., and Tenenbaum, J. B. (2008). Inductive reasoning about causally transmitted properties. Cognition 109, 175192. doi: 10.1016/j.cognition.2008.07.006

Shiffrin, R. M., and Schneider, W. (1977). Controlled and automatic human information processing: II. Perceptual learning, automatic attending and a general theory. Psychol. Rev. 84, 127. doi: 10.1037/0033-295X.84.2.127

Sloutsky, V. M. (2010). From perceptual categories to concepts: what develops? Cogn. Sci. 34, 1244-1286. doi: 10.1111/j.1551-6709.2010. 01129.x

Sloutsky, V. M., and Fisher, A. V. (2008). Attentional learning and flexible induction: how mundane mechanisms give rise to smart behaviors. Child Dev. 79, 639-651. doi: 10.1111/j.1467-8624.2008.01148.x

Sloutsky, V. M., Kloos, H., and Fisher, A. V. (2007). When looks are everything: appearance similarity versus kind information in early induction. Psychol. Sci. 18, 179-185. doi: 10.1111/j.1467-9280.2007.01869.x

Smith, L. B., Colunga, E., and Yoshida, H. (2010). Knowledge as process: contextually cued attention and early word learning. Cogn. Sci. 34, 1287-1314. doi: 10.1111/j.1551-6709.2010.01130.x 
Stanislaw, H., and Todorov, N. (1999). Calculation of signal detection theory measures. Behav. Res. Methods Instrum. Comput. 31, 137-149. doi: 10.3758/BF03207704

Treisman, A. M., and Gelade, G. (1980). A feature-integration theory of attention. Cogn. Psychol. 12, 97-136. doi: 10.1016/0010-0285(80)90005-5

Tversky, A. (1977). Features of similarity. Psychol. Rev. 84, 327-352. doi: 10.1037/0033-295X.84.4.327

Vossel, S., Geng, J. J., and Fink, G. R. (2014). Dorsal and ventral attention systems distinct neural circuits but collaborative roles. Neuroscientist 20, 150-159. doi: $10.1177 / 1073858413494269$

Watanabe, T., and Sasaki, Y. (2015). Perceptual learning: toward a comprehensive theory. Annu. Rev. Psychol. 66, 197-221. doi: 10.1146/annurev-psych-010814015214

Weissman, D. H., and Prado, J. (2012). Heightened activity in a key region of the ventral attention network is linked to reduced activity in a key region of the dorsal attention network during unexpected shifts of covert visual spatial attention. Neuroimage 61, 798-804. doi: 10.1016/j.neuroimage.2012.03.032
Conflict of Interest Statement: The authors declare that the research was conducted in the absence of any commercial or financial relationships that could be construed as a potential conflict of interest.

Received: 08 October 2014; accepted: 13 January 2015; published online: 19 February 2015.

Citation: Hammer R, Sloutsky $V$ and Grill-Spector K (2015) Feature saliency and feedback information interactively impact visual category learning. Front. Psychol. 6:74. doi: 10.3389/fpsyg.2015.00074

This article was submitted to Cognition, a section of the journal Frontiers in Psychology. Copyright (c) 2015 Hammer, Sloutsky and Grill-Spector. This is an open-access article distributed under the terms of the Creative Commons Attribution License (CC BY). The use, distribution or reproduction in other forums is permitted, provided the original author(s) or licensor are credited and that the original publication in this journal is cited, in accordance with accepted academic practice. No use, distribution or reproduction is permitted which does not comply with these terms. 